\title{
Yarkovsky effect detection and updated impact hazard assessment for near-Earth asteroid (410777) 2009 FD
}

\author{
A. Del Vigna ${ }^{1,2}$, J. Roa ${ }^{3}$, D. Farnocchia ${ }^{3}$, M. Micheli ${ }^{4,5}$, D. Tholen ${ }^{6}$, F. Guerra ${ }^{1}$, F. Spoto ${ }^{7}$, and G. B. Valsecchi ${ }^{8,9}$ \\ 1 Space Dynamics Services s.r.l., Via Mario Giuntini, Navacchio di Cascina, Pisa, Italy \\ e-mail: delvigna@mail.dm.unipi.it \\ 2 Dipartimento di Matematica, Università di Pisa, Largo Bruno Pontecorvo 5, Pisa, Italy \\ 3 Jet Propulsion Laboratory, California Institute of Technology, 4800 Oak Grove Drive, Pasadena 91109, CA, USA \\ 4 ESA NEO Coordination Centre, Largo Galileo Galilei, 1, 00044 Frascati, RM, Italy \\ 5 INAF - Osservatorio Astronomico di Roma, Via Frascati, 33, 00040 Monte Porzio Catone, RM, Italy \\ ${ }^{6}$ Institute for Astronomy, University of Hawaii, 2680 Woodlawn Drive, Honolulu, HI 96822, USA \\ 7 Université Côte d'Azur, Observatoire de la Côte d'Azur, CNRS, Laboratoire Lagrange, Boulevard de l'Observatoire, Nice, France \\ 8 IAPS-INAF, Via Fosso del Cavaliere 100, 00133 Roma, Italy \\ 9 IFAC-CNR, Via Madonna del Piano 10, 50019 Sesto Fiorentino, Italy
}

Received 12 June 2019 / Accepted 10 July 2019

\section{ABSTRACT}

\begin{abstract}
Aims. Near-Earth asteroid (410777) 2009 FD is a potentially hazardous asteroid with possible (though unlikely) impacts on Earth at the end of the twenty-second century. The astrometry collected during the 2019 apparition provides information on the trajectory of (410777) by constraining the Yarkovsky effect, which is the main source of uncertainty for future predictions, and improving the impact hazard assessment.

Methods. We included the Yarkovsky effect in the force model and estimated its magnitude from the fit to the optical and radar astrometric data of (410777). We performed the hazard assessment for (410777) over 200 years using two independent approaches: the NEODyS group adopted a generalisation of the Line Of Variations method in a seven-dimensional space, and the JPL team used the Multi-Layer Clustered Sampling technique.

Results. We obtain a $4 \sigma$ detection of the Yarkovsky effect acting on (410777), which corresponds to a semimajor axis drift of $(3.8 \pm 0.9) \times 10^{-3} \mathrm{au} \mathrm{Myr}^{-1}$. In the hazard results of both teams, the main impact possibility in 2185 is ruled out and the only remaining one is in 2190 , but with a probability lower than $10^{-8}$.
\end{abstract}

Key words. minor planets, asteroids: general - celestial mechanics - astrometry

\section{Introduction}

Asteroid (410777) 2009 FD is known to have a non-negligible chance of impacting the Earth in the late twenty-second century. With the optical observations up to 2010 and a purely gravitational model, the resulting orbital solution was very well-constrained and allowed the existence of several Virtual Impactors (VIs, Milani et al. 2005a), in particular between 2185 and 2196, with the highest impact probability predicted for 2185 .

When the optical observations up to April 2014 and the radar Doppler measurement of Arecibo performed on April 7, 2014, were added, the orbital uncertainty had shrunk in such a way that the biggest VI would have been ruled out. However, Spoto et al. (2014) showed that the Yarkovsky effect was a key source of future uncertainty because of the length of the time interval for the impact analysis and because of deep planetary encounters. Unfortunately, the observational data set available in 2014 was not enough to directly detect the Yarkovsky effect from the orbital fit to the astrometry.

To model the Yarkovsky effect, Spoto et al. (2014) therefore relied on the available physical characterisation of (410777) and general properties of the near-Earth asteroid population. When the Yarkovsky effect was accounted for, the 2185 VI could not be ruled out and had an impact probability $I P=2.7 \times 10^{-3}$ and a Palermo Scale $P S=-0.43$, the highest in the risk list of both CLOMON- $2^{1}$ and Sentry ${ }^{2}$. Such a high value of the Palermo Scale was mainly due to the estimated mass of the asteroid. Based on data obtained with the Wide-field Infrared Survey Explorer (WISE), the diameter and the albedo of (410777) were estimated as $(472 \pm 45) \mathrm{m}$ and $(0.010 \pm 0.003)$, respectively (Mainzer et al. 2014 ), yielding a nominal mass of $8.3 \times 10^{10} \mathrm{~kg}$ by assuming a density of $1.5 \mathrm{~g} \mathrm{~cm}^{-3}$, as in Spoto et al. (2014).

The next recomputation of the impact monitoring results by CLOMON-2 and Sentry occurred in early 2016. At that time, the astrometric data set of (410777) included data from two further apparitions: one in late December 2014, and one in OctoberDecember 2015, which also included radar measurements from Arecibo and Goldstone (Naidu et al. 2015). These data led to two main improvements.

- The 2015 radar observations revealed that (410777) is a binary system; the diameter of the two components is roughly $120-180 \mathrm{~m}$ and $60-120 \mathrm{~m}$ (Naidu et al. 2015). These values led to a nominal estimate of $3.2 \times 10^{9} \mathrm{~kg}$ for the mass of

\footnotetext{
1 http://newton. spacedys . com/neodys/index . php?pc=4 . 1 2 http://cneos.jpl.nasa.gov/sentry/
} 
the whole system and in turn to a decrease in the Palermo scale. The updated measurement of the diameter is significantly smaller than previously estimated from data obtained with the Near-Earth Object WISE (NEOWISE; Mainzer et al. 2014) and adopted by Spoto et al. (2014). The inaccuracy of the previous estimate is explained because the WISE measurements of (410777) only correspond to a few of the brightest detections and did not cover the full rotational curve of $(410777)^{3}$. This under-sampling is known to lead to diameter overestimates (Mainzer et al. 2014, Fig. 4).

- The constraint on the Yarkovsky effect from the astrometry became stronger than that from the physical model (see Del Vigna et al. 2018), which is further complicated by the fact that (410777) is a binary (Vokrouhlický et al. 2005). For the impact monitoring results, the set of VIs remained essentially the same as before.

The current astrometric data set available for (410777) includes 39 additional optical observations from the 2019 apparition. We present the updated hazard assessment for (410777), achieved independently with the Line Of Variations (LOV) method, used by NEODyS, and with the Multi-Layer Clustered Sampling (MLCS, Roa \& Farnocchia 2019) technique, adopted by JPL.

\section{Astrometry}

Asteroid (410777) was initially discovered by the La Sagra survey in March 2009, and then linked to precovery observations in the same apparition by the Spacewatch survey, which is consequently credited as discovery site. The available observational arc now extends for ten years and five separate oppositions, from discovery to the latest opposition in 2019. The 2009 and 2014-2015 apparitions are responsible for the majority of the astrometry; the latter also contains all the radar detections.

Most observations collected so far during the 2019 apparition were reported by the Spacewatch team or by serendipitous detections by near-Earth objects surveys. In addition, our group provided observations from ESA's Optical Ground Station (Minor Planet Center code J04) and the University of Hawaii 2.2 meter telescope (Minor Planet Center code T12). For these observations the astrometric uncertainty of each position is available and has been included in the orbit determination process (see Table 1). The formal uncertainties for code T12 include contributions from the astrometric solution and the centroiding on the target asteroid, added in quadrature. Uncertainties caused by variations in the sky transparency, seeing, and tracking of the telescope are much harder to quantify, but can be empirically estimated as $0.05^{\prime \prime}$ primarily in the along-track direction. In general, differential refraction also contributes to the uncertainty (see Tholen \& Farnocchia 2018), although the zenith distance of $18 \mathrm{deg}$ for both the 2019 March 9 and April 4 observations suggests that the amount is at most a few hundredths of an arcsecond. We thus conservatively adopted an uncertainty floor of $0.10^{\prime \prime}$ for these three observations. For the other observations no uncertainty information is available, and we therefore assumed standard weights based on the Vereš et al. (2017) error model.

A search for precovery observations in the image archives of various professional telescopes, executed via the CADC SSOIS interface (Gwyn et al. 2012), did not produce any additional detection. The object was never brighter than $V=24$ at any of the times where suitable images were being exposed by any of the $\simeq 50$ professional instruments covered by the archive.

\footnotetext{
Mainzer and Masiero, priv. comm.
}

Table 1. Observational data of the 2019 apparition, from observatories J04 and T12, with their formal uncertainties.

\begin{tabular}{cccccc}
\hline \hline Date & $\alpha$ & $\delta$ & $\sigma_{\alpha}$ & $\sigma_{\delta}$ & Code \\
\hline $2019-03-06.907914$ & $10: 14: 23.603$ & +173615.77 & $0.30^{\prime \prime}$ & $0.30^{\prime \prime}$ & J04 \\
$2019-03-06.910166$ & $10: 14: 22.988$ & +173618.29 & $0.15^{\prime \prime}$ & $0.15^{\prime \prime}$ & J04 \\
$2019-03-06.912418$ & $10: 14: 22.326$ & +173621.61 & $0.15^{\prime \prime}$ & $0.15^{\prime \prime}$ & J04 \\
$2019-03-09.439509$ & $10: 02: 15.925$ & +183208.44 & $0.036^{\prime \prime}$ & $0.035^{\prime \prime}$ & T12 \\
$2019-03-09.440157$ & $10: 02: 15.723$ & +183209.19 & $0.044^{\prime \prime}$ & $0.044^{\prime \prime}$ & T12 \\
$2019-04-04.264404$ & $07: 32: 22.014$ & +234430.00 & $0.031^{\prime \prime}$ & $0.031^{\prime \prime}$ & T12 \\
\hline
\end{tabular}

Notes. Table columns list calendar date (year, month, and day), right ascension $\alpha$ in hours, minutes, and seconds, declination $\delta$ in degrees, minutes, and seconds, uncertainty of $\alpha$ and $\delta$, and the observatory code.

\section{Detection of the Yarkovsky effect}

For this purpose we modelled the Yarkovsky perturbation using a transverse acceleration

$\mathbf{a}_{t}=\frac{A_{2}}{r^{2}} \hat{\mathbf{t}}$

where $r$ is the heliocentric distance and $A_{2}$ is the dynamical parameter to be estimated from the fit (Farnocchia et al. 2013; Chesley et al. 2016; Del Vigna et al. 2018). Furthermore, the force model we adopted includes the gravitational accelerations of the Sun, the eight planets, and the Moon based on the JPL planetary ephemerides DE431 (Folkner et al. 2014), the perturbations of 16 massive Main-Belt bodies and Pluto, and the Einstein-Infeld-Hoffmann relativistic model (Moyer 2003).

With the weighting scheme described in Sect. 2, the Yarkovsky parameter obtained by the NEODyS group is $A_{2}^{\text {NEODyS }}=(7.3 \pm 1.7) \times 10^{-14} \mathrm{au} \mathrm{d}^{-2}$, which is very consistent with the value $A_{2}^{\mathrm{JPL}}=(7.4 \pm 1.7) \times 10^{-14} \mathrm{au} \mathrm{d}^{-2}$ found by the JPL team. The difference between the two estimates is caused by the fact that the JPL orbit solution assumes a onesecond time uncertainty for each ground-based optical observation, whereas the NEODyS solution does $\operatorname{not}^{4}$. The NEODyS estimate corresponds to a semimajor axis drift $\mathrm{d} a / \mathrm{d} t=(3.8 \pm$ $0.9) \times 10^{-3} \mathrm{au} \mathrm{Myr}^{-1}$. Thus the current astrometry provides a $4 \sigma$ Yarkovsky detection. This distribution of the $A_{2}$ parameter is statistically consistent with a positive Yarkovsky drift: more precisely, the probability of $A_{2}>0$ computed using a Gaussian formalism is $99.998 \%$. The positive value of $A_{2}$ suggests that (410777) is a prograde rotator (Vokrouhlický et al. 2000).

\section{Impact monitoring with the LOV method}

The inclusion of the Yarkovsky effect in the dynamical model results in an initial space with seven dimensions, corresponding to the six orbital parameters and $A_{2}$. The LOV definition of Milani et al. (2005b) can be extended to spaces with dimension greater than six, as was already done in Spoto et al. (2014), with an experimental version the OrbFit software. This capability has undergone testing, and it is now included in the operational version OrbFit 5.0 5 .

The LOV definition remains basically the same: the tangent vector to the LOV at one of its points is the local weak direction, which is the eigenvector of the $7 \times 7$ covariance matrix

\footnotetext{
4 Observations of GPS satellites at T12 on the same nights as the observations of (410777) demonstrate clock accuracy at a level well within the uncertainty assumed by JPL.

5 http://adams.dm.unipi.it/orbfit/
} 
Table 2. Impact monitoring results of asteroid (410777) 2009 FD with a non-gravitational model including the Yarkovsky effect.

\begin{tabular}{cccccc}
\hline \hline Date & $\sigma$ & $\begin{array}{c}\text { Distance } \\
\left(R_{\oplus}\right)\end{array}$ & $\begin{array}{c}\text { Stretching } \\
\left(R_{\oplus}\right)\end{array}$ & $I P$ & $P S$ \\
\hline $2190-03-30.08$ & -4.807 & 0.57 & $1.22 \times 10^{3}$ & $5.56 \times 10^{-9}$ & -7.25 \\
\hline
\end{tabular}

Notes. Table columns list calendar date (year, month, and day) of the potential impact, $\sigma$ value of the VI location along the LOV, distance of the VI trace from the centre of the Earth, local stretching at $\sigma$ (Milani et al. 2005b), probability of Earth impact, and Palermo Scale. The width of the $b$-plane confidence region is $<1 \mathrm{~km}$, thus not reported.

related to the highest eigenvalue. Moreover, cases like (410777), for which the initial confidence region is very small, allow the use of the linear approximation of the LOV: a particular direction $\mathbf{w} \in \mathbb{R}^{7}$ is selected to approximate the LOV with the straight line passing through the nominal solution $\mathbf{x}^{*}$ with direction $\mathbf{w}$. In case of a scattering encounter (Spoto et al. 2014, Sect. 4), w is chosen in such a way that the spread of the corresponding $b$-plane points (Valsecchi et al. 2003) is maximum, so that we can fully capture the different dynamical evolution of the orbits after the scattering encounter along the LOV. In particular, this is obtained by first computing the weak direction on the scattering $b$-plane and then selecting one of its infinitely many preimages in the initial elements space through the semilinear formalism (Milani \& Valsecchi 1999). We sampled the LOV over the interval $|\sigma| \leq \sigma_{\max }=5$ and with a generic completeness level of the VI search $I P^{*}=1 \times 10^{-7}$. This can be achieved with a step-size that is inversely proportional to the probability density along the $\mathrm{LOV}$, resulting in a sampling that is denser around the nominal solution and more sparse towards the LOV tails (Del Vigna et al. 2019). To avoid low resolution in the tail of the distribution, we used a maximum value for the step-size $\Delta \sigma_{\max }=0.01$. This setup led to the computation of 4719 LOV orbits, to propagate with final time in 2250.

The impact monitoring results for (410777) are shown in Table 2. As anticipated, the 2185 impact possibility has disappeared but the 2190 VI still remains, although located in the LOV tails and thus with a low impact probability. The inclusion of the 2019 astrometry and the consequent improvement of the Yarkovsky effect estimate decreased the extent of the LOV projection on the $2185 b$-plane and excluded any impact possibility within $\sigma_{\max }=5$. The VI still exists, but is located at $\sigma \simeq 7$, and the impact probability is negligible. Typically, the effect of a close approach is to separate nearby orbits, thus increasing the uncertainty at subsequent encounters. The increased post-2185 uncertainty allows the existence of a VI in 2190.

\section{Impact monitoring with the MLCS technique}

The JPL impact monitoring analysis was performed using the MLCS technique (Roa \& Farnocchia 2019), which is an efficient alternative to direct Monte Carlo methods. Initially, MLCS generated the first layer of virtual asteroids by randomly drawing 100000 samples from the seven-dimensional normal distribution of the orbital elements and $A_{2}$. Identifying the 20th percentile of samples sorted by closest approach distance provides an interval of $A_{2}$ that contains the values leading to a close approach in 2190. Next, we sampled a second layer containing twice as many points as the previous one. We selected the virtual asteroids for which $A_{2}$ falls within the interval defined in the preceding step, and we propagated them to the 2190 encounter. The

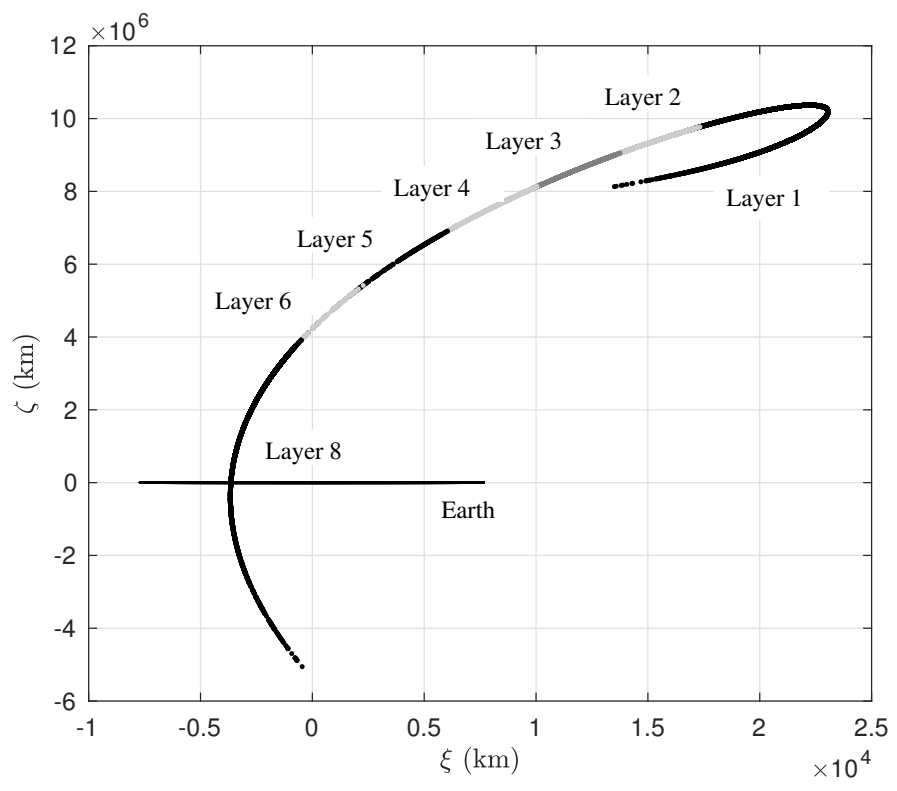

Fig. 1. Distribution of virtual asteroids on the $2190 b$-plane organised in layers of increasing density. Each set of samples satisfies tighter constraints on the value of $A_{2}$ according to the MLCS procedure. The axes use different scales. Layer 7 was omitted for clarity.

20th percentile of this new set produces a reduced $A_{2}$ interval that can be used for further filtering. The process is repeated sequentially until less than a fraction of $10^{-5}$ samples satisfies the condition on $A_{2}{ }^{6}$. In this case, eight layers were required for convergence. The final step consists of sampling $10^{9}$ virtual asteroids and propagating only those that satisfy the $A_{2}$ constraint imposed by the last layer. Figure 1 shows how MLCS iteratively converges to a range of $A_{2}$ that allows an efficient exploration of the final layer. As MLCS advances to the next layer, the samples approach the Earth when mapped to the 2190 $b$-plane.

The distribution of samples in each layer is statistically consistent with the original seven-dimensional distribution in orbital elements and $A_{2}$, and the impact probability and its standard deviation can be computed like in the regular Monte Carlo method. We obtained $I P=(1.5 \pm 0.4) \times 10^{-8}$. The difference in the result relative to the NEODyS one is fully explained by the fact that the JPL orbit solution (JPL 100) assumes a one-second time uncertainty for optical observations, which is not part of the NEODyS orbit determination process. The values of $A_{2}$ compatible with impact trajectories are $A_{2}=(-6.5 \pm 0.4) \times 10^{-15}$ au d ${ }^{2}$, suggesting that an impact is only possible if (410777) were a retrograde rotator.

\section{Estimate of the $\mathbf{2 1 9 0}$ keyhole width and location}

For a pair of resonant returns, a keyhole is one of the preimages of the Earth impact cross section (Chodas 1999). In other words, if an asteroid passes through a keyhole during the first encounter, it will hit the Earth at the subsequent encounter. In our case, we can estimate the width of the keyhole on the $2185 b$-plane for impacts in 2190 using the analytical theory of close encounters, as in Valsecchi et al. (2003). To this end we first computed the

6 This threshold is small enough to efficiently reduce the number of virtual asteroids to propagate while ensuring that enough samples are left to produce a statistically significant result because this threshold is three orders of magnitude greater than the expected impact probability. 


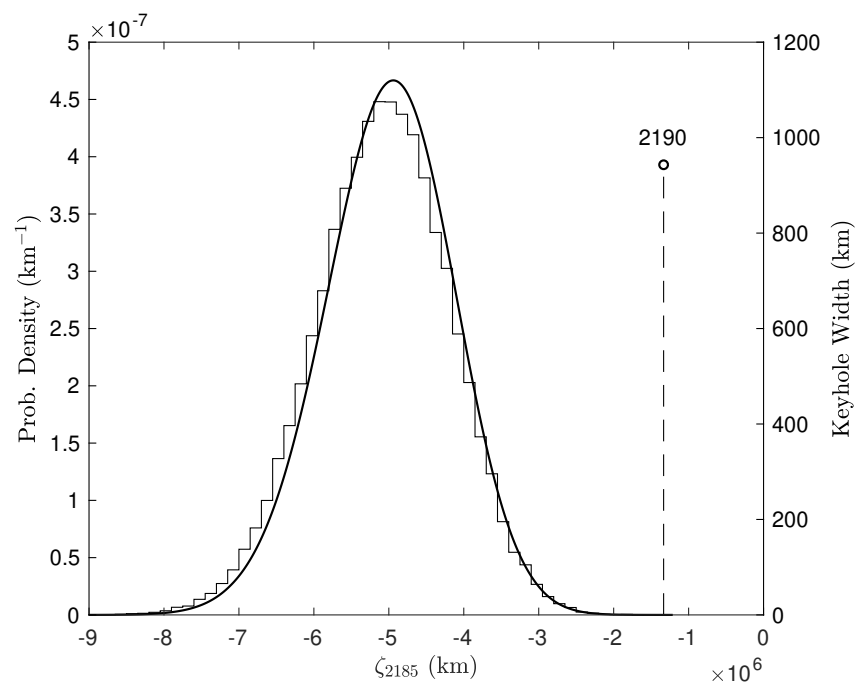

Fig. 2. Graph of the NEODyS non-linear probability density function $p(\zeta)$ on the $2185 b$-plane (bold curve), whose scale is reported on the left vertical axis. The 2190 keyhole is represented by the vertical line segment, which is located at the keyhole centre $\zeta_{k}=-1.333461 \times 10^{6} \mathrm{~km}$ and whose height is equal to the width $w_{k}=943 \mathrm{~km}$, with the scale on the right vertical axis. The histogram represents the distribution based on the virtual asteroids of the first MLCS layer from JPL solution 100.

length of the chord $C$ obtained by intersecting the LOV trace on the $2190 b$-plane with the Earth impact cross section. Then the value of the keyhole width was estimated through the quantity $\partial \zeta^{\prime \prime} / \partial \zeta$, where $(\xi, \zeta)$ and $\left(\xi^{\prime \prime}, \zeta^{\prime \prime}\right)$ are the coordinates on the two pre-encounter $b$-planes, respectively. This derivative can be seen as the factor by which the stretching increases in the time span between the first and the second encounter.

On the $2190 b$-plane, the chord $C$ is contained in the LOV portion between the virtual asteroids bracketing the VI. Let $\left(\xi_{1}^{\prime \prime}, \zeta_{1}^{\prime \prime}\right)$ and $\left(\xi_{2}^{\prime \prime}, \zeta_{2}^{\prime \prime}\right)$ be their coordinates. The length of $C$ is

$\ell_{C}=2 \sqrt{b_{\oplus}^{2}-\frac{\left(\xi_{1}^{\prime \prime} \zeta_{2}^{\prime \prime}-\zeta_{1}^{\prime \prime} \xi_{2}^{\prime \prime}\right)^{2}}{\left(\xi_{2}^{\prime \prime}-\xi_{1}^{\prime \prime}\right)^{2}+\left(\zeta_{2}^{\prime \prime}-\zeta_{1}^{\prime \prime}\right)^{2}}}=2.156 R_{\oplus}$

The derivative $\partial \zeta^{\prime \prime} / \partial \zeta$ depends on the semimajor axis $a$ and the unperturbed geocentric velocity $U$ of (410777) at the 2185 encounter. In our computations, we assumed $a=1.1636 \mathrm{au}$ and $U=16.2 \mathrm{~km} \mathrm{~s}^{-1}$, which are the values corresponding to the virtual asteroid closest to the VI. The $2190 b$-plane is accessible from the $2185 b$-plane through the $4: 5$ mean motion resonance between the asteroid and the Earth. Therefore, after $h=4$ revolutions of the asteroid and $k=5$ revolutions of the Earth, a second close approach takes place. The 4:5 resonance corresponds to the post-encounter semimajor axis $a^{\prime}=\sqrt[3]{k^{2} / h^{2}}=1.1604 \mathrm{au}$. By the equations in Valsecchi et al. (2003, Sect. 4.3) we obtain $\partial \zeta^{\prime \prime} / \partial \zeta \simeq 14.6$, and so the width of the keyhole is

$w_{k} \simeq \frac{\ell_{C}}{\partial \zeta^{\prime \prime} / \partial \zeta}=943 \mathrm{~km}$.

The centre of the keyhole, being the preimage of the midpoint of $C$, is located at $\zeta_{k}=-1.333461 \times 10^{6} \mathrm{~km}$. This point belongs to the LOV portion between the two considered virtual asteroids on the $2185 b$-plane, which are in turn located close to the resonant circle corresponding to the $4: 5$ resonance $^{7}$.

\footnotetext{
7 The locus of points on a given $b$-plane corresponding to a certain mean motion resonance is a circle, with centre on the $\zeta$-axis, as shown in Valsecchi et al. (2003).
}

Figure 2 shows the probability density function $p(\zeta)$ on the $2185 b$-plane, along with the location and width of the keyhole for impacts in 2190 . The position of the keyhole centre on the tail of the probability distribution implies that the associated impact probability is very low. In particular, the impact probability can be estimated as $I P \simeq w_{k} p\left(\zeta_{k}\right) \simeq 5.3 \times 10^{-9}$, which agrees well with the result of Table 2 .

\section{Conclusions}

We presented the new impact monitoring results for asteroid (410777) computed by both the NEODyS and JPL teams after the 2019 apparition. These 2019 astrometric observations extended the observational arc by four years and allowed a $4 \sigma$ detection of the Yarkovsky effect through an orbital fit to the astrometry.

To perform the hazard assessment, we resorted to two independent approaches: the LOV method for NEODyS and the MLCS technique for JPL. Both systems removed the 2185 VI, which was the largest one until the inclusion of the 2019 data. The only remaining VI is the one in 2190 , but because it lies towards the end of the LOV $(|\sigma| \simeq 4.8)$, it has an impact probability $<10^{-8}$, which effectively rules out the corresponding impact.

Acknowledgements. A. Del Vigna and F. Guerra acknowledge support by the company SpaceDyS. Part of this research was conducted at the Jet Propulsion Laboratory, California Institute of Technology, under a contract with NASA. Dave Tholen acknowledges NASA support for his observational work, under the grant No. NNX13AI64G. The work of F. Spoto is supported by the CNES fellowship research programme. This research has made use of data and/or services provided by the International Astronomical Union's Minor Planet Center.

\section{References}

Chesley, S. R., Farnocchia, D., Pravec, P., \& Vokrouhlický, D. 2016, in Asteroids: New Observations, New Models, eds. S. R. Chesley, A. Morbidelli, R. Jedicke, \& D. Farnocchia, IAU Symp., 318, 250

Chodas, P. W. 1999, AAS/Division for Planetary Sciences Meeting Abstracts \#31, 28.04

Del Vigna, A., Faggioli, L., Spoto, F., et al. 2018, A\&A, 617, A61

Del Vigna, A., Milani, A., Spoto, F., Chessa, A., \& Valsecchi, G. B. 2019, Icarus, 321,647

Farnocchia, D., Chesley, S. R., Vokrouhlický, D., et al. 2013, Icarus, 224, 1

Folkner, W. M., Williams, J. G., Boggs, D. H., Park, R. S., \& Kuchynka, P. 2014, Interplanet. Network Prog. Rep., 196, 1

Gwyn, S. D. J., Hill, N., \& Kavelaars, J. J. 2012, PASP, 124, 579

Mainzer, A., Bauer, J., Grav, T., et al. 2014, ApJ, 784, 110

Milani, A., \& Valsecchi, G. B. 1999, Icarus, 140, 408

Milani, A., Chesley, S., Sansaturio, M. E., Tommei, G., \& Valsecchi, G. B. 2005a, Icarus, 173,362

Milani, A., Sansaturio, M., Tommei, G., Arratia, O., \& Chesley, S. R. 2005b, A\&A, 431, 729

Moyer, T. 2003, Formulation for Observed and Computed Values of Deep Space Network Data Types for Navigation, Deep-space Communications and Navigation Series (Wiley-Interscience)

Naidu, S. P., Benner, L. A. M., \& Brozovic, M. 2015, (410777) 2009 FD, Tech. rep. (Central Bureau for Astronomical Telegrams), 4191

Roa, J., \& Farnocchia, D. 2019, Multilayer Clustered Sampling Technique (MLCS) for Near-earth Asteroid Impact Hazard Assessment, 29th SpaceFlight Mechanics Meeting No. 19-260

Spoto, F., Milani, A., Farnocchia, D., et al. 2014, A\&A, 572, A100

Tholen, D. J., \& Farnocchia, D. 2018, AAS/Division for Planetary Sciences Meeting Abstracts, 111.04

Valsecchi, G. B., Milani, A., Gronchi, G. F., \& Chesley, S. R. 2003, A\&A, 408, 1179

Vereš, P., Farnocchia, D., Chesley, S. R., \& Chamberlin, A. B. 2017, Icarus, 296, 139

Vokrouhlický, D., Milani, A., \& Chesley, S. R. 2000, Icarus, 148, 118

Vokrouhlický, D., Čapek, D., Chesley, S. R., \& Ostro, S. J. 2005, Icarus, 179, 128 